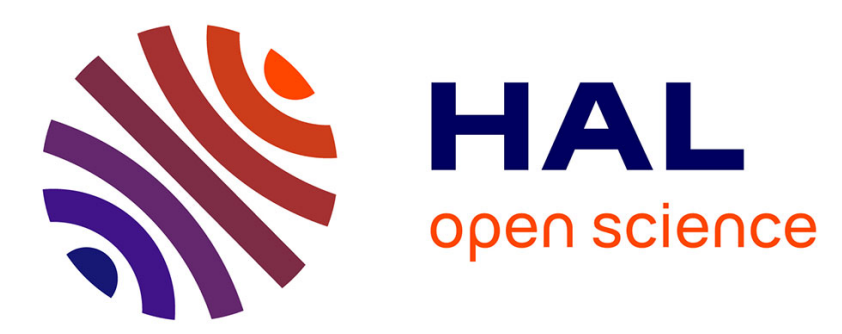

\title{
Lyman- $\alpha$ radiation of a metastable hydrogen beam to measure electric fields
}

\author{
A Lejeune, Laurence Chérigier-Kovacic, F Doveil
}

\section{To cite this version:}

A Lejeune, Laurence Chérigier-Kovacic, F Doveil. Lyman- $\alpha$ radiation of a metastable hydrogen beam to measure electric fields. Applied Physics Letters, 2011, 99, 10.1063/1.3658459 . hal-03534251

\section{HAL Id: hal-03534251 \\ https://hal.science/hal-03534251}

Submitted on 19 Jan 2022

HAL is a multi-disciplinary open access archive for the deposit and dissemination of scientific research documents, whether they are published or not. The documents may come from teaching and research institutions in France or abroad, or from public or private research centers.
L'archive ouverte pluridisciplinaire HAL, est destinée au dépôt et à la diffusion de documents scientifiques de niveau recherche, publiés ou non, émanant des établissements d'enseignement et de recherche français ou étrangers, des laboratoires publics ou privés. 


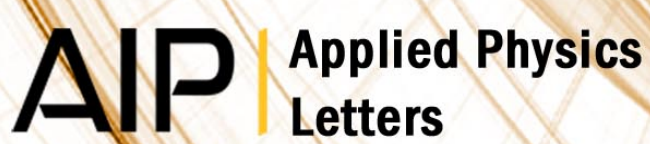

\section{Lyman- $\alpha$ radiation of a metastable hydrogen beam to measure electric fields}

\author{
A. Lejeune, L. Chérigier-Kovacic, and F. Doveil
}

Citation: Appl. Phys. Lett. 99, 181502 (2011); doi: 10.1063/1.3658459

View online: http://dx.doi.org/10.1063/1.3658459

View Table of Contents: http://apl.aip.org/resource/1/APPLAB/v99/i18

Published by the American Institute of Physics.

\section{Related Articles}

Cavity ring-down spectroscopy of the oxygen B-band with absolute frequency reference to the optical frequency comb

J. Chem. Phys. 136, 024201 (2012)

Spectroscopic properties of alkali atoms embedded in Ar matrix

J. Chem. Phys. 135, 174503 (2011)

Spectroscopy of lithium atoms sublimated from isolation matrix of solid $\mathrm{Ne}$

J. Chem. Phys. 135, 134201 (2011)

Optical emission spectroscopy for simultaneous measurement of plasma electron density and temperature in a low-pressure microwave induced plasma

Phys. Plasmas 16, 103501 (2009)

Two-photon resonant second harmonic generation in atomic xeon

J. Chem. Phys. 130, 094305 (2009)

\section{Additional information on Appl. Phys. Lett.}

Journal Homepage: http://apl.aip.org/

Journal Information: http://apl.aip.org/about/about_the_journal

Top downloads: http://apl.aip.org/features/most_downloaded

Information for Authors: http://apl.aip.org/authors

\section{ADVERTISEMENT}

\section{Q \\ DVIDIA. \\ ACCELERATE COMPUTATIONAL CHEMISTRY BY 5X. TRY IT ON A FREE, REMOTELY-HOSTED CLUSTER.}




\title{
Lyman- $\alpha$ radiation of a metastable hydrogen beam to measure electric fields
}

\author{
A. Lejeune, ${ }^{a)}$ L. Chérigier-Kovacic, ${ }^{\text {b) }}$ and F. Doveil ${ }^{\text {c) }}$ \\ Equipe Turbulence Plasma, Case 321, PIIM, UMR6633 CNRS/Université de Provence, campus St. Jérôme, \\ FR-13397 Marseilles cedex 20, France
}

(Received 12 August 2011; accepted 17 October 2011; published online 3 November 2011)

\begin{abstract}
The interaction between a metastable $\mathrm{H}(2 s)$ atomic hydrogen beam and an external electric field leads to the emission of the Lyman- $\alpha$ line. It originates in the Stark mixing of the near-degenerate $2 s_{1 / 2}$ and $2 p_{1 / 2}$ levels separated by the Lamb shift. The quenched radiation proportional to the square of the electric field amplitude is recovered in vacuum by using such an atomic probe beam. We observe the strong enhancement of the signal when the field is oscillating at the Lamb shift frequency. This technique is applied in a plasma, offering an alternative way to measure weak electric fields by direct and non-intrusive means. () 2011 American Institute of Physics. [doi:10.1063/1.3658459]
\end{abstract}

We describe a direct and non-perturbing measurement of electric field with high spatial and temporal resolutions and exceptional sensitivity. It is a critical problem in experimental classical physics and more precisely for diluted fluids and ionized media. For the hydrogenic family, the atomic system possesses a first excited state which contains two nearly degenerate levels $\left(2 s_{1 / 2}, 2 p_{1 / 2}\right)$ with very different lifetimes, separated in energy by the Lamb shift. ${ }^{1}$ As a consequence, the ratio of lifetime differences to energy difference is unusually large for these particles. Weak electric fields as low as $10^{-2}$ $\mathrm{V} / \mathrm{cm}$ for static fields $\left(10^{-3} \mathrm{~V} / \mathrm{cm}\right.$ for oscillating fields) are thus able to mix levels, shorten the $2 s_{1 / 2}$ lifetime, and largely enhance the intensity of the characteristic Lyman- $\alpha$ radiation $(\lambda=121.6 \mathrm{~nm})$ emitted by the $2 s_{1 / 2}$ particles. This effect is several orders of magnitude more sensitive than other Stark processes. The technique presented in this contribution is based on (1) the production and the injection of a metastable hydrogen atom probe beam ${ }^{2}$ into a measurement region and (2) the measurement of the enhanced Lyman- $\alpha$ emission, which is linked to the electric field magnitude.

The fine structure levels $2 s_{1 / 2}$ and $2 p_{1 / 2}$ for hydrogenic atoms and ions are near-degenerate, as was first measured by Lamb. ${ }^{3}$ Contrary to the predictions of the Dirac theory, the $2 s$ state does not have exactly the same energy as the $2 p$ state. It lies higher by a small amount corresponding to a frequency $v_{0}=1057 \mathrm{MHz}$. The degeneracy is removed by small quantum electrodynamic effects taken into account by the radiative corrections. ${ }^{4}$ They are the source of the energy shift called the Lamb shift, which we take advantage of in our experiment to reach a highly sensitive and time resolved electric field measurement. As the $2 s_{1 / 2}$ level is metastable, it is easily transported as long as it stays unperturbed. The lifetime of $2 s_{1 / 2}$ is about $0.14 \mathrm{~s}$, which is very long compared to $1.6 \times 10^{-9} \mathrm{~s}$ for the radiative $2 p_{1 / 2}$ state. However, a transition $2 s_{1 / 2} \rightarrow 1 s_{1 / 2}$ can occur when atoms are sent through a region where an electric field is applied. The quenching of

\footnotetext{
${ }^{a)}$ Present address: ICARE, UPR3021 CNRS-INSIS, 1c Av. de la Recherche Scientifique, 45071 Orléans cedex 2, France. Electronic mail: aurelien. lejeune@cnrs-orleans.fr.

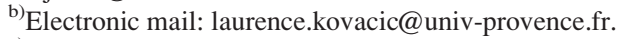

${ }^{c)}$ Electronic mail: fabrice.doveil@univ-provence.fr.
}

the metastable $2 s$ state in the presence of externally applied electric fields leads to the production of a Lyman- $\alpha$ radiation. The intensity is expressed as follows:

$$
I_{\alpha}=\Omega \mathcal{V} n_{2 s} \gamma_{2 s}^{*},
$$

where $\Omega$ is a geometric factor, $\mathcal{V}$ is the interacting volume, $n_{2 s}$ is the $2 s$ metastable hydrogen atom density, and $\gamma_{2 s}^{*}=1 / \tau_{2 s}^{*}$ is the quenched $2 s$ transition rate related to the reduced lifetime. It results from Stark mixing between the $2 s$ and $2 p$ levels. ${ }^{5,6}$ As long as the magnitude of the applied electric field is large enough for the fine structure to be unimportant, the Stark Hamiltonian can be treated like a perturbation of the non relativistic Hamiltonian for the unperturbed hydrogen atom where the $n=2$ level is four times degenerate. The Stark splitting is then linear and partially removes this degeneracy, keeping $\left(2 s_{1 / 2}, 2 p_{1 / 2}\right)$ still degenerate. For lower fields from the limit of $475 \mathrm{~V} / \mathrm{cm}$, the Lamb shift is appreciable in the Stark splitting, and the electric fields are considered as perturbations of the nearly degenerate system $\left(2 s_{1 / 2}, 2 p_{1 / 2}\right)$. Lamb generalized the theory ${ }^{4}$ given by Bethe and Salpeter ${ }^{7}$ to calculate the decay rate $\gamma_{2 s}^{*}$ of the $2 s_{1 / 2}$ level. The time-dependent Schrödinger equation is solved ${ }^{8,9}$ using the perturbation theory when an oscillating electric field of magnitude $E_{0}$ lower than $475 \mathrm{~V} / \mathrm{cm}$ and frequency $\omega / 2 \pi$ is applied to the nearly degenerate system of levels $2 s_{1 / 2}$ and $2 p_{1 / 2}$. The effective decay rate is thus expressed as follows:

$$
\gamma_{2 s}^{*}(\omega)=\left(\frac{3 e a_{0} E_{0}}{\hbar}\right)^{2} \frac{\gamma_{2 p}}{\left(\omega-\omega_{0}\right)^{2}+\gamma_{2 p}^{2} / 4},
$$

where $e$ is the elementary charge, $a_{0}$ is the Bohr radius, $\gamma_{2 p}=1 / \tau_{2 p}$ is the decay rate of the $2 p$ state, $\hbar \omega_{0}$ is the energy difference corresponding to the Lamb shift, and $E_{0}$ is the electric field applied along one direction. We validate in our experiment the quadratic relation of the emission with $E_{0}$ in vacuum for $\omega \rightarrow 0$ (Refs. 10 and 11) and for $\omega \rightarrow \omega_{0}$ as established by Lamb. ${ }^{4}$ At the resonance, for $\omega=\omega_{0}$, we expect to reach a gain of about 440 independently of the field value. This accounts for the theoretical ability to detect small electric fields in a range of $1 \mathrm{mV} / \mathrm{cm}$. The actual experimental resolution depends on the detection system. 
The experimental set-up has been described previously. ${ }^{12}$ A DC hydrogen plasma produced in a magnetic multipole forms the basic source of ions. ${ }^{13}$ The pressure range is from about $10^{-5}$ to $10^{-4} \mathrm{mbar}$. The plasma contains different charged species such as $\mathrm{H}^{+}, \mathrm{H}_{2}^{+}$, and $\mathrm{H}_{3}^{+}$. The ion fractions were measured by mass spectrometry. The ion beam is horizontally extracted (x-axis) and focused through electrostatic lenses separated by ceramics. The wall of the ion source can be biased between 0 and $500 \mathrm{~V}$ to set the kinetic energy of the extracted ion beam. A charge exchange cell containing cesium $(\mathrm{Cs})$ vapor was built from a previously existing device. ${ }^{14}$ It is used to produce the metastable hydrogen atoms from the protons contained in the ion beam through the reaction $\mathrm{H}^{+}+\mathrm{Cs} \rightarrow \mathrm{H}(2 s)+\mathrm{Cs}^{+}$. A $500 \mathrm{eV}$ ion beam corresponds to the maximum cross section of the charge exchange process. ${ }^{15}$ The resulting atomic beam along the $\mathrm{x}$-axis is then injected into a measurement chamber where two horizontal parallel plates separated by $5 \mathrm{~cm}$ are externally biased to create a vertical static or oscillating electric field (z-axis), one plate being simply grounded. The Lyman- $\alpha$ light is collected in a direction perpendicular to the beam (y-axis) by a lithium fluoride (LiF) lens and detected by a vacuum ultraviolet (VUV)-photomultiplier. Spatial resolution is determined by the size of the photocathode and the lens magnification. In order to improve the signal to noise ratio, the non-grounded plate is biased with an amplitude modulation (typ. $1 \mathrm{kHz}$ ), and the modulated signal is detected with a lock-in amplifier. Only the part of the signal due to the field that is synchronized with the biased plate modulation is observed. The measurement chamber allows testing of the beam in vacuum or in a plasma created by a negatively biased filament. For applications in unknown media, the beam extraction can also be modulated.

Circles in Fig. 1 feature the intensity of the Lyman- $\alpha$ radiation in vacuum obtained when there is no cesium along the ion beam path, as a function of the perturbing static voltage applied between the plates. The variation agrees with a quadratic law as shown by the parabolic solid curve for an applied voltage lower than $120 \mathrm{~V}$ (electric field strength lower than $24 \mathrm{~V} / \mathrm{cm}$ since the plate separation is $5 \mathrm{~cm}$ ). Since such a signal was not expected from the ion beam, we tried to vary the ion ratio in the beam to determine the source of this emission. We then observed that this signal was still present when the ion beam contained no $\mathrm{H}_{3}^{+}$at all. In addition, the theory described above for the hydrogenic family

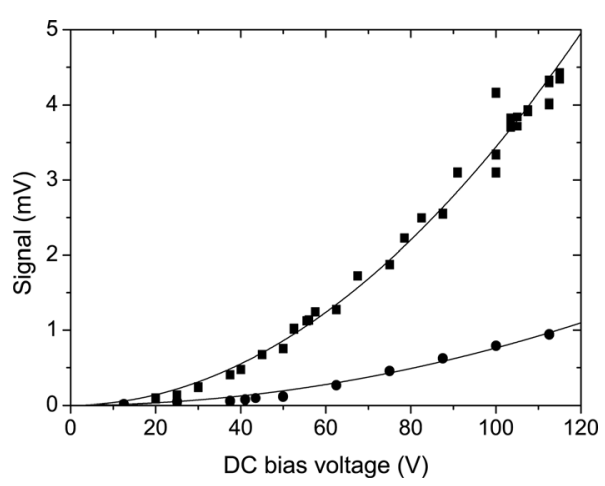

FIG. 1. Quenched Lyman- $\alpha$ emission versus static voltage with (squares) and without (circles) conversion in Cs cell. does not apply to hydrogen ions, since it requires a special energy levels scheme where levels are near-degenerate, which can hardly be found in $\mathrm{H}_{2}^{+}$. As the intensity of the emission being proportional to the gas pressure in the source, we conclude that this radiation is probably emitted by $\mathrm{H}(2 s)$ atoms resulting from the reactions between $\mathrm{H}^{+}$or $\mathrm{H}_{2}^{+}$ions and $\mathrm{H}_{2}$ molecules of the residual gas, which occur along the beam path.

Squares in Fig. 1 represent the intensity of the Lyman- $\alpha$ radiation in vacuum as a function of the perturbing static voltage applied between the plates in the case of a $\mathrm{H}(2 s)$ atom beam is prepared in the cesium cell. The ion source was in the same conditions as previously discussed. As a consequence of the Stark mixing, the variation law is quadratic for an applied static voltage lower than $120 \mathrm{~V}$ (electric field lower than $24 \mathrm{~V} / \mathrm{cm}$ ). As expected, the signal is higher by a factor of 4.5 using cesium.

In order to prove the great capacity of this method to measure weak electric fields, we verified the enhancement predicted by the calculation when the frequency of the applied electric field is resonant with the Lamb shift frequency $\left(\omega \rightarrow \omega_{0}\right)$. We produced a $1 \mathrm{GHz}$ radio frequency (RF) field in our measurement chamber using the biased plate as an emitting antenna. For the lock-in detection, the RF field was still modulated in amplitude at a low frequency around $1 \mathrm{kHz}$. Fig. 2 displays the quadratic law for an oscillating field at the Lamb shift frequency. It also shows that the signal is about 300 times higher than the signal obtained with a constant voltage applied between the plates as in Fig. 1.

This method was applied within a low density DC argon discharge plasma with density $n_{\mathrm{e}} \approx 10^{8} \mathrm{~cm}^{-3}$ created at low pressure $P \approx 10^{-5}$ mbar from a heated filament biased at $-60 \mathrm{~V}$. The profile of the electric field magnitude was obtained by moving the biased plates vertically around the plasma-beam interacting volume. For the lock-in detection, the $\mathrm{H}(2 s)$ atom beam was modulated. Fig. 3 represents the electric field measured both in vacuum (open triangles) and in a plasma (solid triangles) at different positions of the beam between the plates. By applying a constant electric field of $40 \mathrm{~V} / \mathrm{cm}$ in vacuum, we observe the Debye shielding in the presence of the plasma. The electric field is much stronger close to the electrodes due to the formation of plasma sheaths. It falls down to a value slightly smaller than the vacuum value out of the sheaths. This value is not null, most likely due to incompletely neutralized beam space

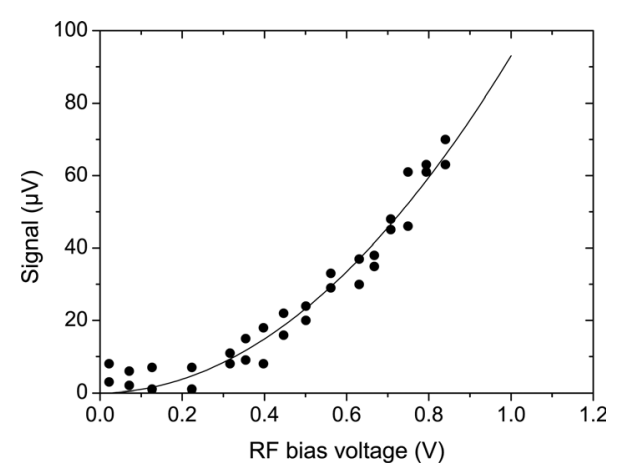

FIG. 2. Quenched Lyman- $\alpha$ emission versus radio frequency ( $1 \mathrm{GHz})$ voltage with a $\mathrm{H}(2 s)$ atom beam. 


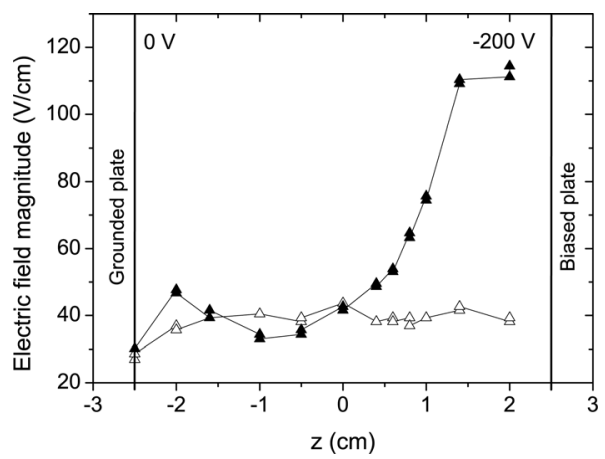

FIG. 3. Electric field measured in vacuum (open triangles) and in a plasma (solid triangles) with a $\mathrm{H}(2 s)$ atom beam.

charge and noise induced by collisions. Measurements reveal boundary effects related to the beam width.

The presented method and results rest on a firm foundation since the technology of $\mathrm{H}(2 s)$ beams and the underlying science are thoroughly established. Through an in-situ electric field calibration of the detection system, these measurements show the ability to non-intrusively measure the absolute values of the electric field. Local magnetic fields could also be measured through the unfolding of the motional $\mathbf{v} \times \mathbf{B}$ field. In addition to vacuum, the investigated medium can be a diluted fluid or a plasma. Indeed, the only non-perturbing space and time resolved method applied in a plasma is based on the Stark splitting for Rydberg states measured by LIF spectroscopy. ${ }^{16}$ In conclusion, the results displayed in this letter open alternative tracks for nonintrusive measurements of electric fields.

The authors dedicate this publication to the late Professor R.A. Stern who inspired this research. The authors are grateful to B. Squizzaro and J.-P. Busso for their technical assistance. They acknowledge the help of G. Bachet and G. Prasad for the conception and construction of the experimental set-up. A. Lejeune benefited from a grant by Ministère de la Recherche.

${ }^{1}$ W. E. Lamb, Jr., Rep. Prog. Phys. 14, 19 (1951).

${ }^{2}$ J. F. Benage, Jr., R. R. Stevens, Jr., and R. A. Stern, Nucl. Instrum. Methods Phys. Res. A 274, 13 (1989).

${ }^{3}$ W. E. Lamb, Jr. and R. C. Retherford, Phys. Rev. 72, 241 (1947).

${ }^{4}$ W. E. Lamb, Jr. and R. C. Retherford, Phys. Rev. 79, 549 (1950).

${ }^{5} \mathrm{C}$. Cohen-Tannoudji, B. Diu, and F. Laloë, Mécanique Quantique, Tome II (Hermann, Paris, 1973).

${ }^{6} \mathrm{~B}$. H. Bransden and C. J. Joachain, Physics of Atoms and Molecules (Longman Scientific and Technical, London, 1983).

${ }^{7}$ H. A. Bethe and E. E. Salpeter, Quantum Mechanics of One and TwoElectron Atoms (Springer, Berlin, 1957).

${ }^{8}$ J. F. Benage, Jr., "Plasma effects on the metastable H(2s) atoms," Ph.D. thesis, University of Colorado, 1986.

${ }^{9}$ A. Lejeune, "Mise au point d'un diagnostic optique non intrusif pour la mesure des micro-fluctuations locales des champs électriques dans les plasmas," Thèse de doctorat, Université de Provence, 2010.

${ }^{10}$ I. A. Sellin, Phys. Rev. A 136, 1245 (1964).

${ }^{11}$ P. Pradel, F. Roussel, A. S. Schlachter, G. Spiess, and A. Valance, Phys. Lett. A 44, 55 (1973).

${ }^{12}$ G. Prasad, G. Bachet, F. Doveil, L. Chérigier, and R. A. Stern, in Proceedings of the Europhysics Conference Abstracts of 1998 ICPP and 25th Conference on Controlled Fusion and Plasma Physics (European Physical Society, Praha, 1998), Vol. 22C.

${ }^{13}$ A. Lejeune, L. Chérigier-Kovacic, F. Doveil, in Proceedings of the Europhysics Conference Abstracts and 35th Conference on Controlled Fusion and Plasma Physics (European Physical Society, Hersonissos, 2008), Vol. 32D, p. 1.186.

${ }^{14}$ M. Bacal, A. Truc, H. J. Doucet, H. Lamain, and M. Chrétien, Nucl. Instrum Methods 114, 407 (1974).

${ }^{15}$ P. Pradel, F. Roussel, A. S. Schlachter, G. Spiess, and A. Valance, Phys. Rev. A 10, 797 (1974).

${ }^{16}$ U. Czarnetzki, D. Luggenhölschler, and H. F. Döbele, Plasma Sources Sci. Technol. 8, 230 (1999). 